\title{
Solvability of fractional boundary value problem with $p$-Laplacian operator at resonance
}

\section{Tengfei Shen, Wenbin Liu* and Xiaohui Shen}

\section{"Correspondence:}

wblium@163.com

College of Sciences, China

University of Mining and

Technology, Xuzhou, 221116

P.R. China

\begin{abstract}
In this paper, a class of multi-point boundary value problems for nonlinear fractional differential equations at resonance with $p$-Laplacian operator is considered. By using the extension of Mawhin's continuation theorem due to $\mathrm{Ge}$, the existence of solutions is obtained, which enriches previous results.

MSC: $34 \mathrm{~A} 08 ; 34 \mathrm{~B} 15$

Keywords: fractional differential equation; boundary value problem; $p$-Laplacian operator; coincidence degree theory; resonance
\end{abstract}

\section{Introduction}

In the recent years, fractional differential equations played an important role in many fields such as physics, electrical circuits, biology, control theory, etc. (see [1-7]). Thus, many scholars have paid more attention to fractional differential equations and gained some achievements (see [8-22]). For example, Wang [21] considered a class of fractional multipoint boundary value problems at resonance by Mawhin's continuation theorem (see [23]):

$$
\left\{\begin{array}{l}
D_{0^{+}}^{\alpha} u(t)=f\left(t, u(t), D_{0^{+}}^{\alpha-1} u(t)\right), \quad t \in(0,1), \text { a.e. } t \in(0,1), \\
u(0)=0, \quad D_{0^{+}}^{\alpha-1} u(1)=\sum_{i=1}^{m} a_{i} D_{0^{+}}^{\alpha-1} u\left(\xi_{i}\right), \\
D_{0^{+}}^{\alpha-2} u(1)=\sum_{i=1}^{m} b_{i} D_{0^{+}}^{\alpha-2} u\left(\eta_{i}\right),
\end{array}\right.
$$

where $1<\alpha \leq 2,0<\xi_{1}<\xi_{2}<\cdots<\xi_{m}<1,0<\eta_{1}<\eta_{2}<\cdots<\eta_{n}<1, \sum_{i=1}^{m} a_{i}=1, \sum_{i=1}^{n} b_{i}=1$, $\sum_{i=1}^{n} b_{i} \eta_{i}=1, D_{0^{+}}^{\alpha}$ is the standard fractional derivative, $f:[0,1] \times \mathbf{R}^{2} \rightarrow \mathbf{R}$ satisfies the Carathéodory condition.

But Mawhin's continuation theorem is not suitable for quasi-linear operators. In [24], Ge and Ren had extended Mawhin's continuation theorem, which was used to deal with more general abstract operator equations. In [25], Pang et al. considered a higher order nonlinear differential equation with a $p$-Laplacian operator at resonance:

$$
\left\{\begin{array}{l}
\left(\varphi_{p}\left(u^{(n-1)}(t)\right)\right)^{\prime}=f\left(t, u(t), \ldots, u^{(n-1)}(t)\right)+e(t), \quad t \in(0,1) \\
u^{(i)}(0)=0, \quad i=1,2, \ldots, n-1, \\
u(1)=\int_{0}^{1} u(s) d g(s)
\end{array}\right.
$$


where $\varphi_{p}(s)=|s|^{p-2} s, p>1, f:[0,1] \times \mathbf{R}^{n} \rightarrow \mathbf{R}^{n}$ and $e:[0,1] \rightarrow \mathbf{R}$ are continuous, $n \geq 2$ is an integer. $g:[0,1] \rightarrow \mathbf{R}$ is a nondecreasing function with $\int_{0}^{1} d g(s)=1$, the integral in the second part of (1.2) is meant in the Riemann-Stieltjes sense.

However, there are few articles which consider the fractional multi-point boundary value problem at resonance with $p$-Laplacian operator and $\operatorname{dim} \operatorname{Ker} M=2$, because $p$-Laplacian operator is a nonlinear operator, and it is hard to construct suitable continuous projectors. In this paper, we will improve and generalize some known results.

Motivated by the work above, our article is to investigate the multi-point boundary value problem at resonance for a class of Riemanne-Liouville fractional differential equations with $p$-Laplacian operator and $\operatorname{dim} \operatorname{Ker} M=2$ by constructing suitable continuous projectors and using the extension of Mawhin's continuation theorem:

$$
\left\{\begin{array}{l}
D_{0^{+}}^{\beta} \varphi_{p}\left(D_{0^{+}}^{\alpha} u(t)\right)=f\left(t, u(t), D_{0^{+}}^{\alpha-2} u(t), D_{0^{+}}^{\alpha-1} u(t), D_{0^{+}}^{\alpha} u(t)\right), \quad t \in(0,1), \\
u(0)=D_{0^{+}}^{\alpha} u(0)=0, \quad u(1)=\sum_{i=1}^{m} a_{i} u\left(\xi_{i}\right), \\
D_{0^{+}}^{\alpha-1} u(1)=\sum_{i=1}^{m} b_{i} D_{0^{+}}^{\alpha-1} u\left(\eta_{i}\right),
\end{array}\right.
$$

where $2<\alpha \leq 3,0<\beta \leq 1,3<\alpha+\beta \leq 4,0<\xi_{1}<\xi_{2}<\cdots<\xi_{m}<1,0<\eta_{1}<\eta_{2}<\cdots<\eta_{m}<1$, $a_{i} \in \mathbf{R}, b_{i} \in \mathbf{R}, 1<m, m \in N, \sum_{i=1}^{m} a_{i} \xi_{i}^{\alpha-1}=1, \sum_{i=1}^{m} a_{i} \xi_{i}^{\alpha-2}=1, \sum_{i=1}^{m} b_{i}=1, \varphi_{p}(s)=|s|^{p-2} s$, $\varphi_{p}(0)=0,1<p, 1 / p+1 / q=1, \varphi_{p}$ is invertible and its inverse operator is $\varphi_{q}, D_{0^{+}}^{\alpha}$ is RiemannLiouville standard fractional derivative, $f:[0,1] \times \mathbf{R}^{4} \rightarrow \mathbf{R}$ is continuous.

In order to investigate the problem, we need to suppose that the following conditions hold:

$$
\Lambda=\Lambda_{1} \Lambda_{4}-\Lambda_{2} \Lambda_{3} \neq 0
$$

where

$$
\begin{aligned}
& \Lambda_{1}=\frac{\Gamma(\alpha)^{q} \Gamma(\alpha q+\beta q-q-\alpha-\beta+2)}{\Gamma(\alpha+\beta)^{q-1} \Gamma(\alpha q+\beta q-q-\beta+2)}\left(1-\sum_{i=1}^{m} a_{i} \xi_{i}^{\alpha q+\beta q-q-\beta+1}\right), \\
& \Lambda_{2}=\frac{\Gamma(\alpha-1)^{q-1} \Gamma(\alpha) \Gamma(\alpha q+\beta q-2 q-\alpha-\beta+3)}{\Gamma(\alpha+\beta-1)^{q-1} \Gamma(\alpha q+\beta q-2 q-\beta+3)}\left(1-\sum_{i=1}^{m} a_{i} \xi_{i}^{\alpha q+\beta q-2 q-\beta+2}\right), \\
& \Lambda_{3}=\frac{\Gamma(\alpha)^{q-1}}{\Gamma(\alpha+\beta)^{q-1}(\alpha q+\beta q-q-\alpha-\beta+2)}\left(1-\sum_{i=1}^{m} b_{i} \eta_{i}^{\alpha q+\beta q-q-\alpha-\beta+2}\right), \\
& \Lambda_{4}=\frac{\Gamma(\alpha-1)^{q-1}}{\Gamma(\alpha+\beta-1)^{q-1}(\alpha q+\beta q-2 q-\alpha-\beta+3)}\left(1-\sum_{i=1}^{m} b_{i} \eta_{i}^{\alpha q+\beta q-2 q-\alpha-\beta+3}\right) .
\end{aligned}
$$

The rest of this article is organized as follows: In Section 2, we give some notations, definitions and lemmas. In Section 3, based on the extension of Mawhin's continuation theorem due to Ge, we establish a theorem on existence of solutions for BVP (1.3).

\section{Preliminaries}

For the convenience of the reader, we present here some necessary basic knowledge and definitions for fractional calculus theory that can be found in the recent literature (see [1, $3,14,21,24,25])$. 
Let $X$ and $Y$ be two Banach spaces with norms $\|\cdot\|_{X}$ and $\|u\|_{Y}$, respectively. A continuous operator

$$
\left.M\right|_{\operatorname{dom} M \cap X}: X \cap \operatorname{dom} M \rightarrow Y
$$

is said to be quasi-linear if

(i) $\operatorname{Im} M:=M(X \cap \operatorname{dom} M)$ is a closed subset of $Y$,

(ii) $\operatorname{Ker} M:=\{u \in X \cap \operatorname{dom} M: M u=0\}$ is linearly homeomorphic to $R^{n}, n<\infty$.

Let $X_{1}=\operatorname{Ker} M$ and $X_{2}$ be the complement space of $X_{1}$ in $X$, then $X=X_{1} \oplus X_{2}$. On the other hand, suppose that $Y_{1}$ is a subspace of $Y$, and $Y_{2}$ is the complement space of $Y_{1}$ in $Y$, so that $Y=Y_{1} \oplus Y_{2}$. Let $P: X \rightarrow X_{1}$ be a projector and $Q: Y \rightarrow Y_{1}$ a semi-projector, and $\Omega \subset X$ an open and bounded set with origin $\theta \in \Omega$. $\theta$ is the origin of a linear space.

Suppose that $N_{\lambda}: \bar{\Omega} \rightarrow Y, \lambda \in[0,1]$ is a continuous operator. Denote $N_{1}$ by $N$. Let $\Sigma_{\lambda}=$ $\left\{u \in \bar{\Omega}: M u=N_{\lambda} u\right\} . N_{\lambda}$ is said to be $M$-compact in $\bar{\Omega}$ if there is an $Y_{1} \subset Y$ with $\operatorname{dim} Y_{1}=$ $\operatorname{dim} X_{1}$ and an operator $R: \bar{\Omega} \times[0,1] \rightarrow X$ continuous and compact such that for $\lambda \in[0,1]$,

$$
\begin{aligned}
& (I-Q) N_{\lambda}(\bar{\Omega}) \subset \operatorname{Im} M \subset(I-Q) Y, \\
& Q N_{\lambda} x=\theta, \quad \lambda \in(0,1) \quad \Leftrightarrow \quad Q N x=\theta, \\
& \left.R(\cdot, \lambda)\right|_{\Sigma_{\lambda}}=\left.(I-P)\right|_{\Sigma_{\lambda}}
\end{aligned}
$$

and $R(\cdot, 0)$ is the zero operator,

$$
M[P+R(\cdot, \lambda)]=(I-Q) N_{\lambda}
$$

Lemma 2.1 (Ge-Mawhin's continuation theorem [24]) Let $\left(X,\|\cdot\|_{X}\right)$ and $\left(Y,\|\cdot\|_{Y}\right)$ be two Banach spaces, and $\Omega \subset X$ an open and bounded nonempty set. Suppose that $M$ : $X \cap \operatorname{dom} M \rightarrow Y$ is a quasi-linear operator $N_{\lambda}: \bar{\Omega} \rightarrow Y, \lambda \in[0,1]$ is $M$-compact in $\bar{\Omega}$. In addition, if

(i) $L u \neq N_{\lambda} u, \forall(u, \lambda) \in(\operatorname{dom} M \cap \partial \Omega) \times(0,1)$,

(ii) $\operatorname{deg}(J Q N, \operatorname{Ker} M \cap \Omega, 0) \neq 0$,

where $J: \operatorname{Im} Q \rightarrow \operatorname{Ker} M$ is a homeomorphism with $J(\theta)=\theta$ and $N=N_{1}$, then the equation $M u=N u$ has at least one solution in $\operatorname{dom} M \cap \bar{\Omega}$.

Definition 2.1 The Riemann-Liouville fractional integral of order $\alpha>0$ of a function $u$ is given by

$$
I_{0^{+}}^{\alpha} u(t)=\frac{1}{\Gamma(\alpha)} \int_{0}^{t}(t-s)^{\alpha-1} u(s) d s
$$

provided the right-hand side integral is pointwise almost everywhere defined on $(0,+\infty)$.

Definition 2.2 The Riemann-Liouville fractional derivative of order $\alpha>0$ of a function $u$ is given by

$$
D_{0^{+}}^{\alpha} u(t)=\frac{1}{\Gamma(n-\alpha)}\left(\frac{d}{d t}\right)^{n} \int_{0}^{t} \frac{u(s)}{(t-s)^{\alpha-n+1}} d s,
$$


provided the right-hand side integral is pointwise everywhere defined on $(0,+\infty)$, where $n=[\alpha]+1$.

Definition 2.3 Let $X$ be a Banach space, and $X_{1} \subset X$ is a subspace. A mapping $Q: X \rightarrow X_{1}$ is a semi-projector if $Q$ satisfies

(i) $Q^{2} x=Q x, \forall x \in X$,

(ii) $Q(\mu x)=\mu Q x, \forall x \in X, \mu \in \mathbf{R}$.

Lemma 2.2 Assume that $u \in C(0,1) \cap L^{1}(0,1)$ with a fractional derivative of order $\alpha>0$ that belongs to $C(0,1) \cap L^{1}(0,1)$. Then

$$
I_{0+}^{\alpha} D_{0+}^{\alpha} u(t)=u(t)+c_{1} t^{\alpha-1}+c_{2} t^{\alpha-2}+\cdots+c_{N} t^{\alpha-N}
$$

for some $c_{i} \in \mathbf{R}, i=1,2, \ldots, N$, where $N=[\alpha]+1$.

Lemma 2.3 Assume that $u(t) \in C[0,1], 0 \leq p \leq q$, then

$$
D_{0^{+}}^{q} I_{0^{+}}^{p} u(t)=I_{0^{+}}^{p-q} u(t) .
$$

Lemma 2.4 Assume that $\alpha \geq 0$, then:

(i) If $\lambda>-1, \lambda \neq \alpha-i, i=1,2, \ldots,[\alpha]+1$, we have that

$$
D_{0^{+}}^{\alpha} t^{\lambda}=\frac{\Gamma(\lambda+1)}{\Gamma(\lambda-\alpha+1)} t^{\lambda-\alpha}
$$

(ii) $D_{0^{+}}^{\alpha} t^{\alpha-i}=0, i=1,2, \ldots,[\alpha]+1$.

In this paper, we take $X=\left\{u \mid u, D_{0^{+}}^{\alpha-2} u, D_{0^{+}}^{\alpha-1} u, D_{0^{+}}^{\alpha} u \in C[0,1]\right\}$ with the norm $\|u\|_{X}=$ $\max \left\{\|u\|_{\infty},\left\|D_{0^{+}}^{\alpha-2} u\right\|_{\infty}\left\|D_{0^{+}}^{\alpha-1} u\right\|_{\infty},\left\|D_{0^{+}}^{\alpha} u\right\|_{\infty}\right\}$, where $\|u\|_{\infty}=\max _{t \in[0,1]}|u(t)|$, and $Y=$ $C[0,1]$ with the norm $\|y\|_{Y}=\|y\|_{\infty}$. By means of the linear functional analysis theory, it is easy to prove that $X$ and $Y$ are Banach spaces, so we omit it.

Define the operator $M: \operatorname{dom} M \rightarrow Y$ by

$$
\begin{aligned}
& M u=D_{0^{+}}^{\beta} \varphi_{p}\left(D_{0^{+}}^{\alpha} u(t)\right), \\
& \operatorname{dom} M=\left\{u \in X \mid D_{0^{+}}^{\beta} \varphi_{p}\left(D_{0^{+}}^{\alpha} u\right) \in Y, u(0)=D_{0^{+}}^{\alpha} u(0)=0,\right. \\
& \left.\quad u(1)=\sum_{i=1}^{m} a_{i} u\left(\xi_{i}\right), D_{0^{+}}^{\alpha-1} u(1)=\sum_{i=1}^{m} b_{i} D_{0^{+}}^{\alpha-1} u\left(\eta_{i}\right)\right\} .
\end{aligned}
$$

Based on the definition of $\operatorname{dom} M$, it is easy to find that $\operatorname{dom} M \neq \varnothing$ such as $u(t)=c t^{\alpha-1} \in$ $\operatorname{dom} M, c \in \mathbf{R}$

Define the operator $N_{\lambda}: X \rightarrow Y, \lambda \in[0,1]$,

$$
N_{\lambda} u(t)=\lambda f\left(t, u(t), D_{0^{+}}^{\alpha-2} u(t), D_{0^{+}}^{\alpha-1} u(t), D_{0^{+}}^{\alpha} u(t)\right), \quad t \in[0,1] .
$$

Then BVP (1.3) is equivalent to the operator equation $M u=N u$, where $N=N_{1}$. 


\section{Main result}

In this section, a theorem on existence of solutions for BVP (1.3) will be given.

Define operators $T_{j}: Y \rightarrow Y, j=1,2$ as follows:

$$
\begin{aligned}
T_{1} y= & \int_{0}^{1}(1-s)^{\alpha-1} \varphi_{q}\left(\frac{1}{\Gamma(\beta)} \int_{0}^{s}(s-\tau)^{\beta-1} y(\tau) d \tau\right) d s \\
& -\sum_{i=1}^{m} a_{i} \int_{0}^{\xi_{i}}\left(\xi_{i}-s\right)^{\alpha-1} \varphi_{q}\left(\frac{1}{\Gamma(\beta)} \int_{0}^{s}(s-\tau)^{\beta-1} y(\tau) d \tau\right) d s, \\
T_{2} y= & \int_{0}^{1} \varphi_{q}\left(\frac{1}{\Gamma(\beta)} \int_{0}^{s}(s-\tau)^{\beta-1} y(\tau) d \tau\right) d s \\
& -\sum_{i=1}^{m} b_{i} \int_{0}^{\eta_{i}} \varphi_{q}\left(\frac{1}{\Gamma(\beta)} \int_{0}^{s}(s-\tau)^{\beta-1} y(\tau) d \tau\right) d s .
\end{aligned}
$$

Let us make some assumptions, which will be used in the sequel.

(H1) There exist nonnegative functions $r, d, e, h, k \in Y$ such that for all $t \in[0,1]$,

$(u, v, w, z) \in \mathbf{R}^{4}$,

$$
|f(t, u, v, w, z)| \leq r(t)+d(t)|u|^{p-1}+e(t)|v|^{p-1}+h(t)|w|^{p-1}+k(t)|z|^{p-1} .
$$

(H2) There exists a constant $A>0$ such that for $u \in \operatorname{dom} M$, if $\left|D_{0^{+}}^{\alpha-1} u(t)\right|>A$ for all $t \in[0,1]$, then

$$
\operatorname{sgn}\left\{D_{0^{+}}^{\alpha-1} u(t)\right\} \frac{1}{\Lambda}\left(\Lambda_{4} T_{1} N u(t)-\Lambda_{3} T_{2} N u(t)\right)>0
$$

or

$$
\operatorname{sgn}\left\{D_{0^{+}}^{\alpha-1} u(t)\right\} \frac{1}{\Lambda}\left(\Lambda_{4} T_{1} N u(t)-\Lambda_{3} T_{2} N u(t)\right)<0 .
$$

(H3) There exists a constant $B>0$ such that for $u \in \operatorname{dom} M$, if $\left|D_{0^{+}}^{\alpha-2} u(t)\right|>B$ for all $t \in[0,1]$, then

$$
\operatorname{sgn}\left\{D_{0^{+}}^{\alpha-2} u(t)\right\} \frac{1}{\Lambda}\left(-\Lambda_{2} T_{1} N u(t)+\Lambda_{1} T_{2} N u(t)\right)>0
$$

or

$$
\operatorname{sgn}\left\{D_{0^{+}}^{\alpha-2} u(t)\right\} \frac{1}{\Lambda}\left(-\Lambda_{2} T_{1} N u(t)+\Lambda_{1} T_{2} N u(t)\right)<0 .
$$

Theorem 3.1 Let $f:[0,1] \times \mathbf{R}^{4} \rightarrow \mathbf{R}$ be continuous and condition (H1)-(H3) hold, then $B V P(1.3)$ has at least one solution, provided that

$$
\frac{1}{\Gamma(\beta+1)}\left(A_{1}\|d\|_{\infty}+\|e\|_{\infty}+\|h\|_{\infty}+\|k\|_{\infty}\right)<1,
$$

where $A_{1}=\frac{1}{\Gamma(\alpha+1)}+\frac{2}{\Gamma(\alpha)}+\frac{7}{2 \Gamma(\alpha-1)}$.

In order to prove Theorem 3.1, we need to prove some lemmas below. 
Lemma 3.1 The operator $M: \operatorname{dom} M \cap X \rightarrow Y$ is quasi-linear.

$$
\begin{aligned}
& \operatorname{Ker} M=\left\{u \in X \mid u(t)=c_{1} t^{\alpha-1}+c_{2} t^{\alpha-2}, c_{1}, c_{2} \in \mathbf{R}\right\}, \\
& \operatorname{Im} M=\left\{y \in Y \mid T_{j} y=0, j=1,2\right\} .
\end{aligned}
$$

Proof Suppose that $u(t) \in \operatorname{dom} M$, by $D_{0^{+}}^{\beta} \varphi_{p}\left(D_{0^{+}}^{\alpha} u(t)\right)=0$, we have

$$
D_{0^{+}}^{\alpha} u(t)=\varphi_{q}\left(c_{0} t^{\beta-1}\right) .
$$

Based on $D_{0^{+}}^{\alpha} u(0)=0$, one has

$$
u(t)=c_{1} t^{\alpha-1}+c_{2} t^{\alpha-2}+c_{3} t^{\alpha-3},
$$

which together with $u(0)=0$ yields that

$$
\operatorname{Ker} M=\left\{u \in X \mid u(t)=c_{1} t^{\alpha-1}+c_{2} t^{\alpha-2}, c_{1}, c_{2} \in \mathbf{R}\right\} .
$$

It is clear that $\operatorname{dim} \operatorname{Ker} M=2$. So, $\operatorname{Ker} M$ is linearly homeomorphic to $\mathbf{R}^{2}$.

If $y \in \operatorname{Im} M$, then there exists a function $u \in \operatorname{dom} M$ such that $y(t)=D_{0^{+}}^{\beta} \varphi_{p}\left(D_{0^{+}}^{\alpha} u(t)\right)$. Based on Lemmas 2.2 and 2.3, we have

$$
\begin{aligned}
& u(t)=I_{0^{+}}^{\alpha} \varphi_{q}\left(I_{0^{+}}^{\beta} y(s)\right)+c_{1} t^{\alpha-1}+c_{2} t^{\alpha-2}, \\
& D_{0^{+}}^{\alpha-1} u(t)=D_{0^{+}}^{\alpha-1} I_{0^{+}}^{\alpha} \varphi_{q}\left(I_{0^{+}}^{\beta} y(s)\right)+c_{1} \Gamma(\alpha),
\end{aligned}
$$

which together with $\sum_{i=1}^{m} a_{i} \xi_{i}^{\alpha-1}=1, \sum_{i=1}^{m} a_{i} \xi_{i}^{\alpha-2}=1$ and $\sum_{i=1}^{m} b_{i}=1$ yields that $T_{j} y(t)=0$, $j=1,2$.

On the other hand, suppose that $y \in Y$ and satisfies (3.3), and let $u(t)=I_{0^{+}}^{\alpha} \varphi_{q}\left(I_{0^{+}}^{\beta} y(t)\right)$, then $u \in \operatorname{dom} M$ and $M u(t)=D_{0^{+}}^{\beta} \varphi_{p}\left(D_{0^{+}}^{\alpha} u(t)\right)=y$, so $y \in \operatorname{Im} M$ and $\operatorname{Im} M:=M(\operatorname{dom} M)$ is a closed subset of $Y$. Thus, $M$ is a quasi-linear operator.

Lemma 3.2 Let $\Omega \subset X$ be an open and bounded set, then $N_{\lambda}$ is M-compact in $\bar{\Omega}$.

Proof Define the continuous projector $P: X \rightarrow X_{1}$ by

$$
P u(t)=\frac{1}{\Gamma(\alpha)} D_{0^{+}}^{\alpha-1} u(0) t^{\alpha-1}+\frac{1}{\Gamma(\alpha-1)} D_{0^{+}}^{\alpha-2} u(0) t^{\alpha-2}, \quad t \in[0,1] .
$$

Define the continuous projector $Q: Y \rightarrow Y_{1}$, by

$$
Q y(t)=\left(Q_{1} y(t)\right) t^{\alpha-1}+\left(Q_{2} y(t)\right) t^{\alpha-2}, \quad t \in[0,1]
$$

where

$$
\begin{aligned}
& Q_{1} y(t)=\varphi_{p}\left(\frac{1}{\Lambda}\left(\Lambda_{4} T_{1} y(t)-\Lambda_{3} T_{2} y(t)\right)\right), \\
& Q_{2} y(t)=\varphi_{p}\left(\frac{1}{\Lambda}\left(-\Lambda_{2} T_{1} y(t)+\Lambda_{1} T_{2} y(t)\right)\right) .
\end{aligned}
$$


Obviously, $X_{1}=\operatorname{Ker} M=\operatorname{Im} P$ and $Y_{1}=\operatorname{Im} Q$. Thus, we have $\operatorname{dim} Y_{1}=\operatorname{dim} X_{1}=2$. For any $y \in Y$, we have

$$
\begin{aligned}
Q_{1}\left(Q_{1} y(t) t^{\alpha-1}\right) & =\varphi_{p}\left(\frac{1}{\Lambda}\left(\Lambda_{4} T_{1}\left(Q_{1} y(t) t^{\alpha-1}\right)-\Lambda_{3} T_{2}\left(Q_{1} y(t) t^{\alpha-1}\right)\right)\right) \\
& =Q_{1} y(t) \varphi_{p}\left(\frac{1}{\Lambda}\left(\Lambda_{4} \Lambda_{1}-\Lambda_{3} \Lambda_{2}\right)\right)=Q_{1} y(t) .
\end{aligned}
$$

Similarly, we can get

$$
Q_{1}\left(Q_{2} y(t) t^{\alpha-2}\right)=0, \quad Q_{2}\left(Q_{1} y(t) t^{\alpha-1}\right)=0, \quad Q_{2}\left(Q_{2} y(t) t^{\alpha-2}\right)=Q_{2} y(t) .
$$

Hence, the map $Q$ is idempotent. Similarly, we can get $Q(\mu y)=\mu Q y$, for all $y \in Y, \mu \in \mathbf{R}$. Thus, $Q$ is a semi-projector. For any $y \in \operatorname{Im} M$, we can get that $Q y=0$ and $y \in \operatorname{Ker} Q$, conversely, if $y \in \operatorname{Ker} Q$, we can obtain that $Q y=0$, that is to say, $y \in \operatorname{Im} M$. Thus, $\operatorname{Ker} Q=$ $\operatorname{Im} M$. Let $\Omega \subset X$ be an open and bounded set with $\theta \in \Omega$, for each $u \in \bar{\Omega}$, we can get $Q\left[(I-Q) N_{\lambda}(u)\right]=0$. Thus, $(I-Q) N_{\lambda}(u) \in \operatorname{Im} M=\operatorname{Ker} Q$. Take any $y \in \operatorname{Im} M$ in the type $y=$ $(y-Q y)+Q y$, since $Q y=0$, we can get $y \in(I-Q) Y$. So, (2.1) holds. It is easy to verify (2.2).

Define $R: \bar{\Omega} \times[0,1] \rightarrow X_{2}$ by

$$
R(u, \lambda)(t)=\frac{1}{\Gamma(\alpha)} \int_{0}^{t}(t-s)^{\alpha-1} \varphi_{q}\left(\frac{1}{\Gamma(\beta)} \int_{0}^{s}(s-\tau)^{\beta-1}\left((I-Q) N_{\lambda} u(\tau)\right) d \tau\right) d s .
$$

By the continuity of $f$, it is easy to get that $R(u, \lambda)$ is continuous on $\bar{\Omega} \times[0,1]$. Moreover, for all $u \in \bar{\Omega}$, there exists a constant $T>0$ such that $\left|I_{0^{+}}^{\beta}(I-Q) N_{\lambda} u(\tau)\right| \leq T$, so, we can easily obtain that $R(\bar{\Omega}, \lambda), D_{0^{+}}^{\alpha-2} R(\bar{\Omega}, \lambda), D_{0^{+}}^{\alpha-1} R(\bar{\Omega}, \lambda)$ and $D_{0^{+}}^{\alpha} R(\bar{\Omega}, \lambda)$ are uniformly bounded. By Arzela-Ascoli theorem, we just need to prove that $R: \bar{\Omega} \times[0,1] \rightarrow X_{2}$ is equicontinuous.

For $u \in \bar{\Omega}, 0<t_{1}<t_{2} \leq 1,2<\alpha \leq 3,0<\beta \leq 1,3<\alpha+\beta \leq 4$, we have

$$
\begin{aligned}
& \left|R(u, \lambda)\left(t_{2}\right)-R(u, \lambda)\left(t_{1}\right)\right| \\
& =\frac{1}{\Gamma(\alpha)} \mid \int_{0}^{t_{2}}\left(t_{2}-s\right)^{\alpha-1} \varphi_{q}\left(I_{0^{+}}^{\beta}\left((I-Q) N_{\lambda} u(\tau)\right)\right) d s \\
& \quad-\int_{0}^{t_{1}}\left(t_{1}-s\right)^{\alpha-1} \varphi_{q}\left(I_{0^{+}}^{\beta}\left((I-Q) N_{\lambda} u(\tau)\right)\right) d s \mid \\
& \leq \frac{\varphi_{q}(L)}{\Gamma(\alpha)}\left(\int_{0}^{t_{1}}\left(\left(t_{2}-s\right)^{\alpha-1}-\left(t_{1}-s\right)^{\alpha-1}\right) d s+\int_{t_{1}}^{t_{2}}\left(t_{2}-s\right)^{\alpha-1} d s\right) \\
& =\frac{\varphi_{q}(T)}{\Gamma(\alpha+1)}\left(t_{2}^{\alpha}-t_{1}^{\alpha}\right), \\
& \left|D_{0^{+}}^{\alpha-2} R(u, \lambda)\left(t_{2}\right)-D_{0^{+}}^{\alpha-2} R(u, \lambda)\left(t_{1}\right)\right| \\
& =\left|\int_{0}^{t_{2}}(t-s) \varphi_{q}\left(I_{0^{+}}^{\beta}\left((I-Q) N_{\lambda} u(\tau)\right)\right) d s-\int_{0}^{t_{1}}(t-s) \varphi_{q}\left(I_{0^{+}}^{\beta}\left((I-Q) N_{\lambda} u(\tau)\right)\right) d s\right| \\
& \leq \varphi_{q}(T)\left(\int_{0}^{t_{1}}\left(t_{2}-s\right)-\left(t_{1}-s\right) d s+\int_{t_{1}}^{t_{2}}\left(t_{2}-s\right) d s\right) \\
& =\frac{\varphi_{q}(T)}{2}\left(t_{2}^{2}-t_{1}^{2}\right)
\end{aligned}
$$


and

$$
\begin{aligned}
& \left|D_{0^{+}}^{\alpha-1} R(u, \lambda)\left(t_{2}\right)-D_{0^{+}}^{\alpha-1} R(u, \lambda)\left(t_{1}\right)\right| \\
& =\left|\int_{0}^{t_{2}} \varphi_{q}\left(I_{0^{+}}^{\beta}\left((I-Q) N_{\lambda} u(\tau)\right)\right) d s-\int_{0}^{t_{1}} \varphi_{q}\left(I_{0^{+}}^{\beta}\left((I-Q) N_{\lambda} u(\tau)\right)\right) d s\right| \\
& \leq \varphi_{q}(T)\left(t_{2}-t_{1}\right) .
\end{aligned}
$$

Since $t^{\alpha}$ is uniformly continuous on $[0,1]$, so, $R(\bar{\Omega}, \lambda), D_{0^{+}}^{\alpha-2} R(\bar{\Omega}, \lambda)$ and $D_{0^{+}}^{\alpha-1} R(\bar{\Omega}, \lambda)$ are equicontinuous. Similarly, we can get that $I_{0^{+}}^{\beta}\left((I-Q) N_{\lambda} u(\tau)\right) \subset C[0,1]$ is equicontinuous. Considering that $\varphi_{q}(s)$ is uniformly continuous on $[-T, T]$, we have that $D_{0^{+}}^{\alpha} R(\bar{\Omega}, \lambda)=$ $I_{0^{+}}^{\beta}\left((I-Q) N_{\lambda}(\bar{\Omega})\right)$ is also equicontinuous. So, we can obtain that $R: \bar{\Omega} \times[0,1] \rightarrow X_{2}$ is compact.

For each $u \in \Sigma_{\lambda}$, we have $D_{0^{+}}^{\beta} \varphi_{p}\left(D_{0^{+}}^{\alpha} u(t)\right)=N_{\lambda}(u(t)) \in \operatorname{Im} M$. Thus,

$$
\begin{aligned}
R(u, \lambda)(t) & =\frac{1}{\Gamma(\alpha)} \int_{0}^{t}(t-s)^{\alpha-1} \varphi_{q}\left(\frac{1}{\Gamma(\beta)} \int_{0}^{s}(s-\tau)^{\beta-1}\left((I-Q) N_{\lambda} u(\tau)\right) d \tau\right) d s \\
& =\frac{1}{\Gamma(\alpha)} \int_{0}^{t}(t-s)^{\alpha-1} \varphi_{q}\left(\frac{1}{\Gamma(\beta)} \int_{0}^{s}(s-\tau)^{\beta-1} D_{0^{+}}^{\beta} \varphi_{p}\left(D_{0^{+}}^{\alpha} u(\tau)\right) d \tau\right) d s
\end{aligned}
$$

which together with $D_{0^{+}}^{\alpha} u(0)=u(0)=0$ yields that

$$
R(u, \lambda)(t)=u(t)-\frac{1}{\Gamma(\alpha)} D_{0^{+}}^{\alpha-1} u(0) t^{\alpha-1}-\frac{1}{\Gamma(\alpha-1)} D_{0^{+}}^{\alpha-2} u(0) t^{\alpha-2}=(I-P) u(t)
$$

It is easy to verify that $R(u, 0)(t)$ is the zero operator. So, (2.3) holds. Besides, for any $u \in \bar{\Omega}$,

$$
\begin{aligned}
M & {[P u+R(u, \lambda)](t) } \\
= & M\left[\frac{1}{\Gamma(\alpha)} \int_{0}^{t}(t-s)^{\alpha-1} \varphi_{q}\left(\frac{1}{\Gamma(\beta)} \int_{0}^{s}(s-\tau)^{\beta-1}\left((I-Q) N_{\lambda} u(\tau)\right) d \tau\right) d s\right. \\
& \left.\quad+\frac{1}{\Gamma(\alpha)} D_{0^{+}}^{\alpha-1} u(0) t^{\alpha-1}+\frac{1}{\Gamma(\alpha-1)} D_{0^{+}}^{\alpha-2} u(0) t^{\alpha-2}\right] \\
= & (I-Q) N_{\lambda} u(t),
\end{aligned}
$$

which implies (2.4). So, $N_{\lambda}$ is $M$-compact in $\bar{\Omega}$.

Lemma 3.3 Suppose that (H1), (H2) hold, then the set

$$
\Omega_{1}=\left\{u \in \operatorname{dom} M \backslash \operatorname{Ker} M \mid M u=N_{\lambda} u, \lambda \in(0,1)\right\}
$$

is bounded.

Proof By Lemma 2.2, for each $u \in \operatorname{dom} M$, we have

$$
u(t)=I_{0^{+}}^{\alpha} D_{0^{+}}^{\alpha} u(t)+c_{1} t^{\alpha-1}+c_{2} t^{\alpha-2}+c_{3} t^{\alpha-3} .
$$


Combined with $u(0)=0$, we get $c_{3}=0$. Thus,

$$
\begin{aligned}
& u(t)=I_{0^{+}}^{\alpha} D_{0^{+}}^{\alpha} u(t)+c_{1} t^{\alpha-1}+c_{2} t^{\alpha-2}, \\
& D_{0^{+}}^{\alpha-1} u(t)=I_{0^{+}}^{1} D_{0^{+}}^{\alpha} u(t)+c_{1} \Gamma(\alpha), \\
& D_{0^{+}}^{\alpha-2} u(t)=I_{0^{+}}^{2} D_{0^{+}}^{\alpha} u(t)+c_{1} \Gamma(\alpha) t+c_{2} \Gamma(\alpha-1) .
\end{aligned}
$$

By simple calculation, we get

$$
\begin{aligned}
& c_{1}=\frac{1}{\Gamma(\alpha)}\left(D_{0^{+}}^{\alpha-1} u(t)-\int_{0}^{t} D_{0^{+}}^{\alpha} u(s) d s\right), \\
& c_{2}=\frac{1}{\Gamma(\alpha-1)}\left(D_{0^{+}}^{\alpha-2} u(t)-\int_{0}^{t}(t-s) D_{0^{+}}^{\alpha-1} u(s) d s-\left(D_{0^{+}}^{\alpha-1} u(t)-\int_{0}^{t} D_{0^{+}}^{\alpha} u(s) d s\right) t\right) .
\end{aligned}
$$

Take any $u \in \Omega_{1}$, then $N u \in \operatorname{Im} M=\operatorname{Ker} Q$ and $Q N u=0$. It follows from (H2) and (H3) that there exist $\varepsilon_{1}, \varepsilon_{2} \in[0,1]$ such that $\left|D_{0^{+}}^{\alpha-1} u\left(\varepsilon_{1}\right)\right| \leq A,\left|D_{0^{+}}^{\alpha-2} u\left(\varepsilon_{2}\right)\right| \leq B$. Thus,

$$
\begin{aligned}
& D_{0^{+}}^{\alpha-1} u(t)=D_{0^{+}}^{\alpha-1} u\left(\varepsilon_{1}\right)+\int_{\varepsilon_{1}}^{t} D_{0^{+}}^{\alpha} u(t) d t, \\
& D_{0^{+}}^{\alpha-2} u(t)=D_{0^{+}}^{\alpha-2} u\left(\varepsilon_{2}\right)+\int_{\varepsilon_{2}}^{t} D_{0^{+}}^{\alpha-1} u(t) d t, \\
& \left\|D_{0^{+}}^{\alpha-1} u\right\|_{\infty} \leq A+\left\|D_{0^{+}}^{\alpha} u\right\|_{\infty} \\
& \left\|D_{0^{+}}^{\alpha-2} u\right\|_{\infty} \leq B+\left\|D_{0^{+}}^{\alpha-1} u\right\|_{\infty} \leq A+B+\left\|D_{0^{+}}^{\alpha} u\right\|_{\infty} .
\end{aligned}
$$

So, we get

$$
\begin{aligned}
\left|c_{1}\right| & \leq \frac{1}{\Gamma(\alpha)}\left(\left\|D_{0^{+}}^{\alpha-1} u\right\|_{\infty}+\left\|D_{0^{+}}^{\alpha} u\right\|_{\infty}\right) \leq \frac{1}{\Gamma(\alpha)}\left(A+2\left\|D_{0^{+}}^{\alpha} u\right\|_{\infty}\right), \\
\left|c_{2}\right| & \leq \frac{1}{\Gamma(\alpha-1)}\left(\left\|D_{0^{+}}^{\alpha-2} u\right\|_{\infty}+\frac{3}{2}\left\|D_{0^{+}}^{\alpha-1} u\right\|_{\infty}+\left\|D_{0^{+}}^{\alpha} u\right\|_{\infty}\right) \\
& \leq \frac{1}{\Gamma(\alpha-1)}\left(\frac{5 A}{2}+B+\frac{7}{2}\left\|D_{0^{+}}^{\alpha} u\right\|_{\infty}\right), \\
\|u\|_{\infty} & \leq A_{1}\left\|D_{0^{+}}^{\alpha} u\right\|_{\infty}+B_{1},
\end{aligned}
$$

where $A_{1}=\frac{1}{\Gamma(\alpha+1)}+\frac{2}{\Gamma(\alpha)}+\frac{7}{2 \Gamma(\alpha-1)}, B_{1}=\frac{A}{\Gamma(\alpha)}+\frac{5 A}{2 \Gamma(\alpha-1)}+\frac{B}{\Gamma(\alpha-1)}$.

Based on $D_{0^{+}}^{\alpha} u(0)=0$, we have

$$
\varphi_{p}\left(D_{0^{+}}^{\alpha} u(t)\right)=\lambda I_{0^{+}}^{\beta} N u(t) .
$$

From $(\mathrm{H} 1)$ and $\lambda \in(0,1)$, we have

$$
\begin{aligned}
& \left|\varphi_{p}\left(D_{0^{+}}^{\alpha} u(t)\right)\right| \\
& \quad \leq \frac{1}{\Gamma(\beta)} \int_{0}^{t}(t-s)^{\beta-1}\left|f\left(s, u(s), D_{0^{+}}^{\alpha-2} u(t), D_{0^{+}}^{\alpha-1} u(s), D_{0^{+}}^{\alpha} u(s)\right)\right| d s \\
& \quad \leq \frac{1}{\Gamma(\beta)} \int_{0}^{t}(t-s)^{\beta-1}\left(r(s)+d(s)|u(s)|^{p-1}+e(s)\left|D_{0^{+}}^{\alpha-2} u(s)\right|^{p-1}\right.
\end{aligned}
$$




$$
\begin{aligned}
& \left.+h(s)\left|D_{0^{+}}^{\alpha-1} u(s)\right|^{p-1}+k(s)\left|D_{0^{+}}^{\alpha} u(s)\right|^{p-1}\right) d s \\
\leq & \frac{1}{\Gamma(\beta+1)}\left(\|r\|_{\infty}+\|d\|_{\infty}\|u\|_{\infty}^{p-1}+\|e\|_{\infty}\left\|D_{0^{+}}^{\alpha-2} u\right\|_{\infty}^{p-1}\right. \\
& \left.+\|h\|_{\infty}\left\|D_{0^{+}}^{\alpha-1} u\right\|_{\infty}^{p-1}+\|k\|_{\infty}\left\|D_{0^{+}}^{\alpha} u\right\|_{\infty}^{p-1}\right),
\end{aligned}
$$

which together with $\left|\varphi_{p}\left(D_{0^{+}}^{\alpha} u(t)\right)\right|=\left|D_{0^{+}}^{\alpha} u(t)\right|^{p-1}$, we can get

$$
\begin{aligned}
\left\|D_{0^{+}}^{\alpha} u\right\|_{\infty}^{p-1} \leq & \frac{1}{\Gamma(\beta+1)}\left(\|r\|_{\infty}+\|d\|_{\infty}\|u\|_{\infty}^{p-1}+\|e\|_{\infty}\left\|D_{0^{+}}^{\alpha-2} u\right\|_{\infty}^{p-1}\right. \\
& \left.+\|h\|_{\infty}\left\|D_{0^{+}}^{\alpha-1} u\right\|_{\infty}^{p-1}+\|k\|_{\infty}\left\|D_{0^{+}}^{\alpha} u\right\|_{\infty}^{p-1}\right) \\
\leq & \frac{1}{\Gamma(\beta+1)}\left(\|r\|_{\infty}+\|d\|_{\infty}\left(A_{1}\left\|D_{0^{+}}^{\alpha} u\right\|_{\infty}+B_{1}\right)+\|e\|_{\infty}\left(2 A+\left\|D_{0^{+}}^{\alpha} u\right\|_{\infty}\right)^{p-1}\right. \\
& \left.+\|h\|_{\infty}\left(A+\left\|D_{0^{+}}^{\alpha} u\right\|_{\infty}\right)^{p-1}+\|k\|_{\infty}\left\|D_{0^{+}}^{\alpha} u\right\|_{\infty}^{p-1}\right) .
\end{aligned}
$$

In view of (3.1), we can obtain that there exists a constant $M_{1}>0$ such that

$$
\begin{aligned}
& \left\|D_{0^{+}}^{\alpha} u\right\|_{\infty} \leq M_{1}, \quad\left\|D_{0^{+}}^{\alpha-1} u\right\|_{\infty} \leq A+M_{1}:=M_{2}, \\
& \left\|D_{0^{+}}^{\alpha-2} u\right\|_{\infty} \leq 2 A+M_{1}:=M_{3}, \quad\|u\|_{\infty} \leq A_{1} M_{1}+B_{1}:=M_{4} .
\end{aligned}
$$

Thus, we have

$$
\|u\|_{X}=\max \left\{\|u\|_{\infty},\left\|D_{0^{+}}^{\alpha-2} u\right\|_{\infty},\left\|D_{0^{+}}^{\alpha-1} u\right\|_{\infty},\left\|D_{0^{+}}^{\alpha} u\right\|_{\infty}\right\} \leq \max \left\{M_{1}, M_{2}, M_{3}, M_{4}\right\}:=M .
$$

So, $\Omega_{1}$ is bounded.

Lemma 3.4 Suppose that $(\mathrm{H} 2)$ holds, then the set

$$
\Omega_{2}=\{u \mid u \in \operatorname{Ker} M, N u \in \operatorname{Im} M\}
$$

\section{is bounded.}

Proof For each $u \in \Omega_{2}$, we have that $u(t)=c_{1} t^{\alpha-1}+c_{2} t^{\alpha-2}, c_{1}, c_{2} \in \mathbf{R}$ and $Q N u=0$. It follows from (H2) and (H3) that there exists an $\varepsilon_{1}, \varepsilon_{2} \in[0,1]$ such that $\left|D_{0^{+}}^{\alpha-1} u\left(\varepsilon_{1}\right)\right| \leq A$, $\left|D_{0^{+}}^{\alpha-2} u\left(\varepsilon_{2}\right)\right| \leq A$, which implies that $\left|c_{1}\right| \leq \frac{A}{\Gamma(\alpha)}$ and $\left|c_{2}\right| \leq \frac{A+B}{\Gamma(\alpha-1)}$. So, $\Omega_{2}$ is bounded.

Define the isomorphism $J^{-1}: \operatorname{Ker} M \rightarrow \operatorname{Im} Q$ by $J^{-1}\left(c_{1} t^{\alpha-1}+c_{2} t^{\alpha-2}\right)=c_{1} t^{\alpha-1}+c_{2} t^{\alpha-2}$, $c_{1}, c_{2} \in \mathbf{R}, t \in[0,1]$. In fact, for each $c_{1}, c_{2} \in R$, suppose that $\left(Q_{1} y(t), Q_{2} y(t)\right)=\left(c_{1}, c_{2}\right)$, we have

$$
\left\{\begin{array}{l}
\Lambda_{4} T_{1} y(t)-\Lambda_{3} T_{2} y(t)=\Lambda \varphi_{q}\left(c_{1}\right):=\widetilde{c_{1}}, \\
-\Lambda_{2} T_{1} y(t)+\Lambda_{1} T_{2} y(t)=\Lambda \varphi_{q}\left(c_{2}\right):=\widetilde{c_{2}},
\end{array}\right.
$$

where $\widetilde{c_{1}}, \widetilde{c_{2}} \in \mathbf{R}$, by the condition $\Lambda_{4} \Lambda_{1}-\Lambda_{2} \Lambda_{3} \neq 0$, there exists a unique solution for (3.4), which is $\left(T_{1} y(t), T_{2} y(t)\right)=\left(m_{1}, m_{2}\right), m_{1}, m_{2} \in \mathbf{R}$. Now, we will prove that there exists $y \in Y$ such that $\left(T_{1} y(t), T_{2} y(t)\right)=\left(m_{1}, m_{2}\right)$. Based on $y(t) \in C[0,1]$, we choose $y(t)=D_{0^{+}}^{\beta} \bar{y}(t)$, 
where $\bar{y}(t)=\varphi_{p}\left(l_{1} t^{(\alpha+\beta-1)(q-1)}+l_{2} t^{(\alpha+\beta-2)(q-1)}\right), l_{1}=\frac{\Delta_{1} \Gamma(\alpha)^{q-1}}{\Gamma(\alpha+\beta)^{q-1}}, l_{2}=\frac{\Delta_{2} \Gamma(\alpha-1)^{q-1}}{\Gamma(\alpha+\beta-1)^{q-1}}, \Delta_{1}=\frac{m_{1} \Lambda_{4}-m_{2} \Lambda_{2}}{\Lambda}$, $\Delta_{2}=\frac{m_{2} \Lambda_{1}-m_{1} \Lambda_{3}}{\Lambda}, t \in[0,1]$, which together with $2<\alpha \leq 3,0<\beta \leq 1,3<\alpha+\beta \leq 4, q>1$, we have $(\alpha+\beta-1)(q-1)>0$ and $(\alpha+\beta-2)(q-1)>0$. So, we have $\bar{y}(0)=0$. Thus, we can obtain that

$$
I_{0^{+}}^{\beta} y(t)=I_{0^{+}}^{\beta} D_{0^{+}}^{\beta} \bar{y}(t)=\bar{y}(t)+c t^{\beta-1},
$$

where $c \in \mathbf{R}$, which together with $\bar{y}(0)=0$, we have $c=0$. So, we have

$$
\left\{\begin{aligned}
T_{1} y(t) & =\int_{0}^{1}(1-s)^{\alpha-1} \varphi_{q}(\bar{y}(s)) d s-\sum_{i=1}^{m} a_{i} \int_{0}^{\xi_{i}}\left(\xi_{i}-s\right)^{\alpha-1} \varphi_{q}(\bar{y}(s)) d s \\
& =\Delta_{1} \Lambda_{1}+\Delta_{2} \Lambda_{2}=m_{1}, \\
T_{2} y(t) & =\int_{0}^{1} \varphi_{q}(\bar{y}(s)) d s-\sum_{i=1}^{m} b_{i} \int_{0}^{\eta_{i}} \varphi_{q}(\bar{y}(s)) d s=\Delta_{1} \Lambda_{3}+\Delta_{2} \Lambda_{4}=m_{2} .
\end{aligned}\right.
$$

Thus, there exists $y \in Y$ such that $\left(T_{1} y(t), T_{2} y(t)\right)=\left(m_{1}, m_{2}\right)$. So $J^{-1}: \operatorname{Ker} L \rightarrow \operatorname{Im} Q$ is well defined.

Lemma 3.5 Suppose that the first part of $(\mathrm{H} 3)$ holds, then the set

$$
\Omega_{3}=\left\{u \in \operatorname{Ker} M \mid \lambda J^{-1} u+(1-\lambda) Q N u=0, \lambda \in[0,1]\right\}
$$

is bounded.

Proof For each $u \in \Omega_{3}$, we can get that $u(t)=c_{1} t^{\alpha-1}+c_{2} t^{\alpha-2}, c_{1}, c_{2} \in \mathbf{R}$. By the definition of the set $\Omega_{3}$, we can obtain that

$$
\lambda\left(c_{1} t^{\alpha-1}+c_{2} t^{\alpha-2}\right)+(1-\lambda)\left(Q_{1} N\left(c_{1} t^{\alpha-1}+c_{2} t^{\alpha-2}\right) t^{\alpha-1}+Q_{2} N\left(c_{1} t^{\alpha-1}+c_{2} t^{\alpha-2}\right) t^{\alpha-2}\right)=0 .
$$

Thus,

$$
\begin{aligned}
& \lambda c_{1}+(1-\lambda) \varphi_{p}\left(\frac{1}{\Lambda}\left(\Lambda_{4} T_{1} N\left(c_{1} t^{\alpha-1}+c_{2} t^{\alpha-2}\right)-\Lambda_{3} T_{2} N\left(c_{1} t^{\alpha-1}+c_{2} t^{\alpha-2}\right)\right)\right)=0, \\
& \lambda c_{2}+(1-\lambda) \varphi_{p}\left(\frac{1}{\Lambda}\left(-\Lambda_{2} T_{1} N\left(c_{1} t^{\alpha-1}+c_{2} t^{\alpha-2}\right)+\Lambda_{1} T_{2} N\left(c_{1} t^{\alpha-1}+c_{2} t^{\alpha-2}\right)\right)\right)=0 .
\end{aligned}
$$

If $\lambda=0$, then $\Omega_{3}$ is bounded because of the first part of (H2) and (H3). If $\lambda=1$, we get $c_{1}=c_{2}=0$, obviously, $\Omega_{3}$ is bounded. If $\lambda \in(0,1)$, by the first part of $(\mathrm{H} 2)$ and (3.5), we can obtain that $\left|c_{1}\right| \leq \frac{A}{\Gamma(\alpha)}$, by the first part of (H3) and (3.6), we have $\left|c_{2}\right| \leq \frac{A+B}{\Gamma(\alpha-1)}$. So, $\Omega_{3}$ is bounded.

Remark 3.1 If the second part of (H3) holds, then the set

$$
\Omega_{3}^{\prime}=\left\{u \in \operatorname{Ker} M \mid-\lambda J^{-1} u+(1-\lambda) Q N u=0, \lambda \in[0,1]\right\}
$$

is bounded. 
Proof of Theorem 3.1 Assume that $\Omega$ is a bounded open set of $X$ with $\bigcup_{i=1}^{3} \bar{\Omega}_{i} \cup \Omega_{3}^{\prime} \subset \Omega$. By Lemmas 3.1 and 3.2, we can obtain that $M: \operatorname{dom} M \cap X \rightarrow Y$ is quasi-linear, and $N_{\lambda}$ is $M$-compact on $\bar{\Omega}$. By the definition of $\Omega$, we have

$$
\begin{aligned}
& L u \neq N_{\lambda} u, \quad \forall(u, \lambda) \in(\operatorname{dom} M \cap \partial \Omega) \times(0,1), \\
& H(u, \lambda)= \pm \lambda J^{-1}(u)+(1-\lambda) Q N(u) \neq 0, \quad(\partial \Omega \cap \operatorname{Ker} M) \times[0,1] .
\end{aligned}
$$

Thus, by the homotopic property of degree, we can get

$$
\begin{aligned}
\operatorname{deg}(U Q N, \Omega \cap \operatorname{Ker} M, 0) & =\operatorname{deg}(H(\cdot, 0), \Omega \cap \operatorname{Ker} M, 0) \\
& =\operatorname{deg}(H(\cdot, 1), \Omega \cap \operatorname{Ker} M, 0) \\
& =\operatorname{deg}( \pm I, \Omega \cap \operatorname{Ker} M, 0) \neq 0 .
\end{aligned}
$$

So Lemma 2.1 is satisfied, and $M u=N u$ has at least one solution in $\operatorname{dom} M \cap \bar{\Omega}$. Namely, BVP (1.3) have at least one solution in the space $X$.

\section{Competing interests}

The authors declare that they have no competing interests.

\section{Authors' contributions}

The authors contributed equally in this article. All authors read and approved the final manuscript.

\section{Acknowledgements}

The authors are grateful to those who gave useful suggestions about the original manuscript. This research is supported by the National Natural Science Foundation of China (No. 11271364) and the Fundamental Research Funds for the Central Universities (2010LKSX09).

Received: 1 January 2013 Accepted: 2 September 2013 Published: 07 Nov 2013

\section{References}

1. Kilbas, AA, Srivastava, HM, Trujillo, JJ: Theory and Applications of Fractional Differential Equations. Elsevier, Amsterdam (2006)

2. Sabatier, J, Agrawal, OP, Machado, JAT: Advances in Fractional Calculus: Theoretical Developments and Applications in Physics and Engineering. Springer, Dordrecht (2007)

3. Samko, SG, Kilbas, AA, Marichev, Ol: Fractional Integrals and Derivatives: Theory and Applications. Gordon \& Breach, New York (1993)

4. Magin, R: Fractional calculus models of complex dynamics in biological tissues. Comput. Math. Appl. 59, 1586-1593 (2010)

5. Metzler, R, Klafter, J: Boundary value problems for fractional diffusion equations. Physica A 278, 107-125 (2000)

6. Mainardi, F: Fractional diffusive waves in viscoelastic solids. In: Wegner, JL, Norwood, FR (eds.) Nonlinear Waves in Solids, pp. 93-97. ASME, Fairfield (1995)

7. Bai, J, Feng, X: Fractional-order anisotropic diffusion for image denoising. IEEE Trans. Image Process. 16, 2492-2502 (2007)

8. Agarwal, RP, O'Regan, D, Stanek, S: Positive solutions for Dirichlet problems of singular nonlinear fractional differential equations. J. Math. Anal. Appl. 371, 57-68 (2010)

9. Kosmatov, N: A boundary value problem of fractional order at resonance. Electron. J. Differ. Equ. 2010, 135 (2010)

10. Jafari, H, Gejji, VD: Positive solutions of nonlinear fractional boundary value problems using Adomian decomposition method. Appl. Math. Comput. 180, 700-706 (2006)

11. Bai, Z: Solvability for a class of fractional $m$-point boundary value problem at resonance. Comput. Math. Appl. 62, 1292-1302 (2011)

12. Bai, Z: On solutions of some fractional $m$-point boundary value problems at resonance. Electron. J. Qual. Theory Differ. Equ. 2010, 37 (2010)

13. Bai, Z, Zhang, Y: Solvability of fractional three-point boundary value problems with nonlinear growth. Appl. Math. Comput. 218, 1719-1725 (2011)

14. Zhang, Y, Bai, Z: Existence of positive solutions for nonlinear fractional three-point boundary value problem at resonance. Appl. Math. Comput. 36, 417-440 (2011)

15. Hu, Z, Liu, W: Solvability for fractional order boundary value problem at resonance. Bound. Value Probl. 2011, 20 (2011)

16. Rui, W: Existence of solutions of nonlinear fractional differential equations at resonance. Electron. J. Qual. Theory Differ. Equ. 2012, 66 (2012) 
17. Wang, G, Liu, W, Yang, J, Zhu, S, Zheng, T: The existence of solutions for a fractional $2 m$-point boundary value problems. J. Appl. Math. (2012). doi:10.1155/2012/841349

18. Wang, W: Solvability for a coupled system of fractional differential equations at resonance. Nonlinear Anal. 13 2285-2292 (2012)

19. Zhou, H, Kou, C, Xie, F: Existence of solutions for fractional differential equations with multi-point boundary conditions at resonance on a half-line. Electron. J. Qual. Theory Differ. Equ. 2011, 27 (2011)

20. Chen, $Y$, Tang, $X$ : Solvability of sequential fractional order multi-point boundary value problems at resonance. Appl. Math. Comput. 218, 7638-7648 (2012)

21. Wang, J: The existence of solutions to boundary value problems of fractional differential equations at resonance. Nonlinear Anal. 74, 1987-1994 (2011)

22. Chen, T, Liu, W, Hu, Z: A boundary value problem for fractional differential equation with $p$-Laplacian operator at resonance. Nonlinear Anal. 75, 3210-3217 (2012)

23. Mawhin, J: Topological Degree and Boundary Value Problems for Nonlinear Differential Equations in Topological Methods for Ordinary Differential Equations. Lecture Notes in Math., vol. 1537, pp. 74-142 (1993)

24. Ge, W, Ren, J: An extension of Mawhin's continuation theorem and its application to boundary value problems with a p-Laplacian. Nonlinear Anal. 58, 477-488 (2004)

25. Pang, $\mathrm{H}, \mathrm{Ge}, \mathrm{W}$, Tian, $\mathrm{M}$ : Solvability of nonlocal boundary value problems for ordinary differential equation of higher order with a p-Laplacian. Comput. Math. Appl. 56, 127-142 (2008)

10.1186/1687-1847-2013-295

Cite this article as: Shen et al.: Solvability of fractional boundary value problem with $p$-Laplacian operator at resonance. Advances in Difference Equations 2013, 2013:295

\section{Submit your manuscript to a SpringerOpen ${ }^{\circ}$ journal and benefit from:}

- Convenient online submission

- Rigorous peer review

- Immediate publication on acceptance

- Open access: articles freely available online

- High visibility within the field

- Retaining the copyright to your article 